\title{
Impact of Educational Program on Knowledge, Attitude and Practice of Prisoners, Officers and Soldiers at Menoufia Governorate Prison, Egypt
}

\author{
WESAM S. MORAD, M.D.* and REDA ABD EL-LATEF, M.D.** \\ The Department of Epidemiology \& Preventive Medicine, National Liver Institute* and \\ The Department of Public Health \& Community Medicine, Faculty of Medicine**, Menoufia University, Menoufia, Egypt
}

\begin{abstract}
Background: Prisons are considered facilities liable of high risk of infectious disease. Investigations carried out in prisons around the world have shown a high prevalence of blood borne hepatitis viruses. As there are few studies aboutblood borne hepatitis viruses knowledge among prisoners and security personnel. Those carried with other populations, point out that the lack of knowledge about the illness is one of the main barriers to the perception of the symptoms, early diagnosis, treatment adherence and cure.
\end{abstract}

Aim of Study: To estimate the level of knowledge, attitudes and practice related to $\mathrm{HBV}$ and $\mathrm{HCV}$ infections before and after an educational intervention among security personnel and prisoners.

Patients and Methods: Self-administered questionnaire was used to assess KAP of HBV and HCV infection of 324 prisoners and 68 security personals pre and post educational intervention. SPSS (Version 20) was used for analysis of data.

Results: The mass media were the major source of information $(44.5 \%)$. Regarding knowledge and attitude about $\mathrm{HBV}$ and $\mathrm{HCV}$ infection, highly significant improvement ( $p$ $<0.001$ ) was revealedamong both security personnel and prisoners. Regarding practice, there was no improvement in post-intervention practice of prisoner $(p>0.05)$; however there was improvement in the practice of security personnel $(p$ $<0.001$ ). Knowledge and attitude scores of security personnel were evident irrespective of socio-demographic characteristics and history of hepatitis infections. Security personnel who were of urban residence had significantlybetter pre-intervention practice $(p=0.006)$. Overall KAP scores among prisonerswere evident irrespective of socio-demographic characteristics. HBV \& HCV infected prisoners $(p<0.001)$ were significantly more knowledgeable about these viruses before educational intervention.

Conclusions: We are reporting inadequate level of HBV and HCV awareness among the studied groups. The study confirms the necessity of continuous periodic health education programs as well as immunization programs, infectious disease

Correspondence to: Dr. Wesam S. Morad,

E-Mail: wesammorad@yahoo.com screening, treatment, and promotion of living conditions in prisons.

Key Words: Prisoners - Security personnel $-H C V-H B V-$ Knowledge - Attitude - Practice.

\section{Introduction}

GLOBALLY, many studies on blood born hepatitis viruses (HBV \& HCV) have been undertaken by various government and nongovernmental organizations among the general public. There are certain high risk groups in well-defined but restricted settings who are usually left out from the interventions they deserve especially in developing countries. Inmates of prisons are example of this left out population [1].

The prevention of blood-borne pathogen transmission is a high priority for the Ministry of Health and Population (MOHP) in Egypt [2]. Despite the availability of several reports concerning antiHepatitis C Virus (HCV) sero-positivity among apparently healthy Egyptian populations, little attention has been paid to HBV and HCV burden of Egyptian prisoners.

Hepatitis C is a worldwide problem, hepatitis $\mathrm{C}$ virus is a major cause of both acute and chronic hepatitis. The World Health Organization (WHO) estimates about $3 \%$ of the world's population has been infected with $\mathrm{HCV}$ and that there are more than 170 million chronic carriers who are at risk of developing liver cirrhosis and/or liver cancer [3].

Egypt has the largest epidemic of hepatitis C virus in the world. The recently released Egyptian Demographic Health Survey (EDHS) tested a rep- 
resentative sample of the entire country for $\mathrm{HCV}$ antibody. Over 11000 individuals were tested. The overall prevalence positive for antibody to $\mathrm{HCV}$ was $14.7 \%$ [4]

Hepatitis B is a serious and common infectious disease of the liver affecting millions of people throughout the world. It is estimated that 350 million individuals worldwide are infected with the virus, which cause 620.000 deaths worldwide each year. Egypt is considered an area of intermediate endemicity for the virus as the prevalence of HBV chronic carriers among adults in the general population ranged from $2 \%$ to $7 \%$ [5].

Prisons are known for connection of high risk of infection diseases [6] as a result of possibility of transmission of infections in prisons surroundings [7]. Persons with inferior health status are main representatives among those who have contact with criminal juristic system [8].

Investigations carried out in correctional facilities around the world have shown a high prevalence of blood borne hepatitis viruses [9]. Among highrisk environments, prisons are well-known establishments for spreading of blood-borne viruses. Those who use illicit drugs or engage in sex work are often incarcerated. In addition, during imprisonment, prisoners often engage in unprotected sex, syringe/needle sharing, or sharing of other paraphernalia (spoon, water) [10], tattooing and scarification are also common [11].

Lack of awareness of the risk of HBV and its consequences are recognized as a major deterrent to immunization among HBV high risk groups [12]

Within the prisons, prison officers potentially have a vital role in maintaining a secure and healthy living environment for the inmates. Besides performing daily security checks and search procedures, a prison officer has responsibilities, such as providing appropriate care and support for inmates and making sure that inmates have access to professional help when needed. Ideally, prison officers must be able to establish and maintain a positive working relationship with inmates, balancing authority with care and understanding [13]

The guidance suggests that as well as improving services for prisoners, ongoing educational program to improve awareness of the risks of $\mathrm{HBV}$ and HCV should be provided to staff in prison and youth offender. This would not only help to reduce the stigma of BBVs across the whole prison estate but would enable staff to take necessary precautions to protect themselves from the risk of occupational infection. As rates of BBVs are higher amongst prisoners than the general population, prisons can be considered an environment at elevated risk of infection [14]

\section{Subjects and Methods}

Participants in the study were categorized into: Prisoners and security personnel. The current study was conducted on 324 prisoners which were categorized into 246 males and 78 females out of 750 eligible prisoners captured in Shebin El-Kom Public Prison (Menoufia Governorate, Egypt) From January 2016 till January 2018. The involved personnel were selected randomly by systematic random sample technique (data from prison list). And also, 68 security personal out of 80 ones working in prison were included in the study which were categorized into 10 policemen, 25 soldiers, 6 jailers, 14 prison employee and 13 prison health care workers. They have been selected randomly upon the instructions of the prison authorities and their work shift time.

Study design:

An intervention design with pre-and posteducation assessment of KAP about HBV and HCV infection was completed among the selected prisoners as well as security personnel working in Shebin El-Kom Public Prison (Menoufia Governorate, Egypt). All participants completed both preand post-intervention questionnaires. The KAP of HBV and HCV infection were assessed using a structured questionnaire completed immediately before and after the educational intervention for prisoners and immediately before and after 6 months from education intervention for security personnel. The study obtained all required ethical approvals from the Ethical Committees of Faculty of Medicine (Menoufia University), Media and Public Relations Department at the Ministry of the Interior, National Security Sector, and the Prison Service Directorate of Health Care. An oral consent was obtained from all participants.

\section{Questionnaire:}

The self-administered questionnaire was developed to assess the KAP of HBV and HCV infection among the study participants and the questions were based on previous surveys with similar objectives [15]. Additionally, the validity of the questionnaire was tested after Arabic translation. The final versionof the questionnaire had a good indicator for reliability (as indicated by Alpha Cronbach test value of 0.82 for knowledge and 0.77 for 
attitude and practice). Sections of the administered questionnaire included personal and sociodemographic characteristics, general health status, and source of previous knowledge and KAP of $\mathrm{HBV}$ and $\mathrm{HCV}$ infection which included (22 questions to asses knowledge, 11 question for attitude and 10 question for practice).

\section{Educational intervention:}

It was established according to the standard principles of designing and implementing a health education program [16]. The educational intervention was provided through; lectures about 16 lectures each session lasted 3 hours and included only 20-25 new participants, distributing educational leaflets (including HBV facts, risk factors, hazard to population, misconceptions and misunderstanding of $\mathrm{HBV}$ and $\mathrm{HCV}$, preventiontools and how to deal with an infected person) and granting booklet providing detailed information about viral hepatitis $\mathrm{B}$ and $\mathrm{C}$.

\section{Statistical analysis:}

Data were presented using descriptive statistics in the form of frequencies and percentages for categorical data overall KAP score was the sum of correct responses to the 43 questions. Two points was given for the correct answer, one point for to some extend and zero for the wrong answer. KAP score and its components' scores were treated as non-parametric data and compared between preand post-intervention using Wilcoxon signed rank test. Satisfactory knowledge, positive attitude and good practice is considered if their score were $>50 \%$. Both pre-and post-intervention KAP scores and their components' scores were compared across socio-demographic characteristics using chi-square test or Fisher exact test as appropriate. All $p$-values were two-tailed. $p$-value $<0.05$ was considered as significant. SPSS software (Version 20.0) SPSS Inc., Chicago, U.S. was used for all statistical analyses.

\section{Results}

$77.9 \%$ of security personnel and $75.9 \%$ of the studied prisoners were male, $61.8 \%$ of security personnel and $67.3 \%$ of prisoners were $<40$ years, the majority of security personnel $(86.8 \%)$ and of prisoners $(73.8 \%)$ were married, the majority of prisoners $(67 \%)$ were of rural residence while the majority of security personnel $(63.2 \%)$ were of urban residence, $67.3 \%$ of the studied prisoners could not read and write, $67.7 \%$ of security personnel had $<10$ years service duration at Shebin El-Kom Public Prison and $83.6 \%$ of the studied prisoners spent $<10$ years at this prison (Table 1 ). The most common sources reported for such knowledge were TV/Radio (44.5\%), health services $(24.8 \%)$, other sources like family and friends (24.2\%), school and college (6.5\%) Fig. (1). The study results showed marked improvement of overall knowledge and attitude after educational intervention $[p$-value $<0.001]$; however, there was no improvement in practice of prisoners $(p>0.05)$ (Table 2). Only $38.2 \%$ of the studied security personnel had satisfied level of knowledge toward viral hepatitis which had been improved into $98.5 \%$ of them were with satisfied level of knowledge. $70.6 \%$ of studied security personnel had positive attitude toward viral hepatitis prior to health education program which improved into $100 \%$. Percentages of studied security personnel with good practice prior to health education program were $64.7 \%$ which improved into $94.1 \%$ (Table 2). However, only $1.9 \%$ had satisfied level of knowledge toward viral hepatitis which had been improved into 67.3 of studied prisoners were with satisfied level of knowledgeafter implementation of health education program. Only $16 \%$ of studied prisoners had positive attitude toward viral hepatitis prior to health education program which improved into $79 \%$. Percentages of studied prisoners with good practice prior to health education program were $5.6 \%$. But this percentage did not changed after implementation of health education program (Table 2). Regarding knowledge score component of security personnel, $39.7 \%$ of them knew different mode of HBV \& HCV transmission, 29.4\% knew clinical picture and $39.7 \%$ knew about preventive and control measures which had been improved into $97.1 \%, 100 \%, 97.1 \%$ respectively after implementation of health education program Fig. (2). Regarding knowledge score component of prisoners, only $3.7 \%$ of them knew mode of transmission and preventive and control measures, $4 \%$ knew clinical picture which had been improved into $86.7 \%, 31.2 \%, 42.9 \%$ respectively Fig. (3). In the current work, there was no significant association between all participants and their sociodemographic data regarding their overall knowledge and attitude score about hepatitis B and C (Tables $3,4)$, however there was significant association between practice score of security personnel and their residence (75\% urban verse $25 \%$ rural) before educational intervention (Table 3). Pre-intervention KAP scores of security personnel were not significantly associated ( $p$-value $>0.05$ ) with previous history of HBV and or HCV infections, however HBV \& HCV infected prisoners $(p<0.001)$ were significantly more knowledgeable about these viruses before educational intervention (Table 5). 
Source of information

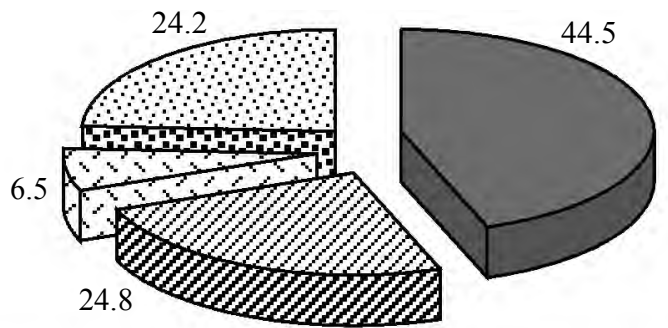

MASS media $\$$ School and collage

Health services

Fig. (1): The main sources of information about hepatitis B \& $\mathrm{C}$ among the studied group before intervention.

Knowledge score pre and post test of security personnel

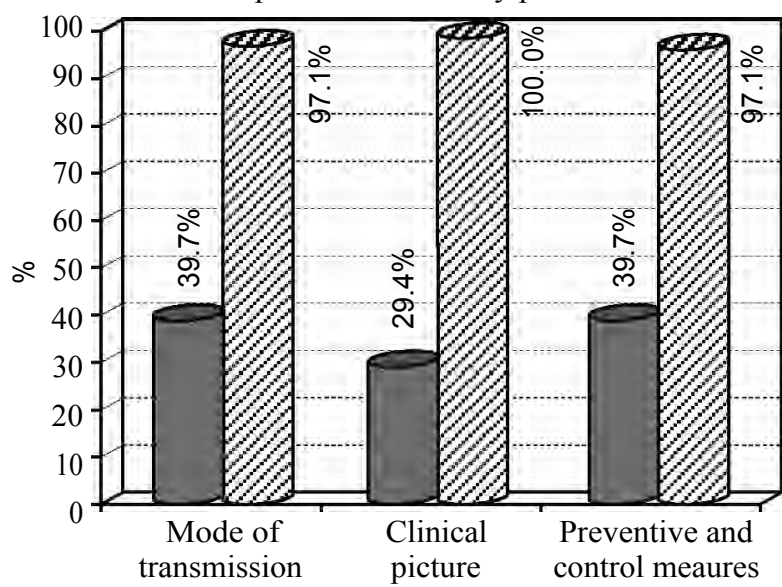

Satisfactory knowledge about

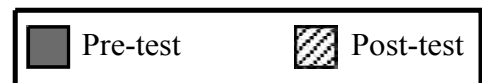

Fig. (2): Knowledge scores of security personnel about HBV and HCV before and after application of health education program.
Knowledge score pre \& post test of the studied prisoners

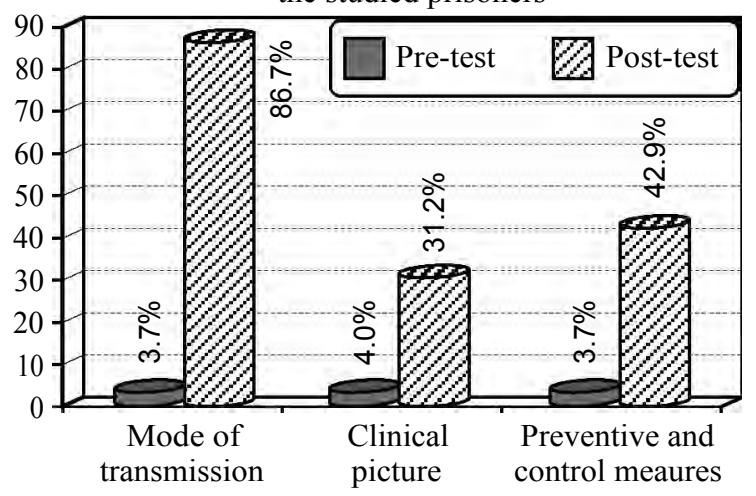

Satisfactory knowledge about

Fig. (3): Knowledge scores of the studied prisoners about HBV and HCV before and after application of health education program.

Table (1): Socio demographic criteria among the studied groups.

\begin{tabular}{|c|c|c|c|c|}
\hline \multirow{2}{*}{$\begin{array}{l}\text { Socio } \\
\text { demographic } \\
\text { data }\end{array}$} & \multicolumn{2}{|c|}{$\begin{array}{l}\text { The studied prisoners } \\
\mathrm{N}=324\end{array}$} & \multicolumn{2}{|c|}{$\begin{array}{c}\text { Security personne } \\
\mathrm{N}=68\end{array}$} \\
\hline & No & $\%$ & No & $\%$ \\
\hline \multicolumn{5}{|l|}{ Age groups: } \\
\hline$<40$ & 218 & 67.3 & 42 & 61.8 \\
\hline$>40$ & 106 & 32.7 & 26 & 38.2 \\
\hline \multicolumn{5}{|l|}{ Sex: } \\
\hline Male & 246 & 75.9 & 53 & 77.9 \\
\hline Female & 78 & 24.1 & 15 & 22.1 \\
\hline \multicolumn{5}{|l|}{ Marital status: } \\
\hline Married & 239 & 73.8 & 59 & 86.8 \\
\hline Unmarried & 85 & 26.2 & 9 & 13.2 \\
\hline \multicolumn{5}{|l|}{ Education: } \\
\hline Illiterate & 218 & 67.3 & & \\
\hline Educated & 106 & 32.7 & 68 & 100 \\
\hline \multicolumn{5}{|l|}{ Residence: } \\
\hline Rural & 217 & 67 & 25 & 36.8 \\
\hline Urban & 107 & 23 & 43 & 63.2 \\
\hline \multicolumn{5}{|l|}{ Work duration: } \\
\hline$<10$ years & & & 46 & 67.7 \\
\hline$>10$ years & & & 22 & 32.4 \\
\hline \multicolumn{5}{|c|}{ Imprisonment duration: } \\
\hline$<10$ years & 270 & 83.6 & & \\
\hline$>10$ years & 53 & 16.4 & & \\
\hline
\end{tabular}

Table (2): Assessment of knowledge, attitude and practice (pre and post-test) among studied security personal and prisoners.

\begin{tabular}{|c|c|c|c|c|c|c|c|c|c|c|}
\hline \multirow{3}{*}{ KAP } & \multicolumn{5}{|c|}{ KAP of security personnel } & \multicolumn{5}{|c|}{ KAP of prisoners } \\
\hline & \multicolumn{2}{|c|}{ Pre $(n=68)$} & \multicolumn{2}{|c|}{ Post $(n=68)$} & \multirow{2}{*}{$\begin{array}{c}p^{-} \\
\text {value }\end{array}$} & \multicolumn{2}{|c|}{$\operatorname{Pre}(n=324)$} & \multicolumn{2}{|c|}{ Post $(n=324)$} & \multirow{2}{*}{$\begin{array}{c}p^{-} \\
\text {value }\end{array}$} \\
\hline & No & $\%$ & No & $\%$ & & No & $\%$ & No & $\%$ & \\
\hline \multicolumn{11}{|l|}{ Knowledge: } \\
\hline $\begin{array}{l}\text { Satisfactory } \\
\text { Unsatisfactory }\end{array}$ & $\begin{array}{l}26 \\
42\end{array}$ & 38.2 & 67 & $\begin{array}{l}98.5 \\
15\end{array}$ & 0.001 & 6 & $\begin{array}{l}1.9 \\
981\end{array}$ & 218 & 67.3 & 0.001 \\
\hline \multicolumn{11}{|l|}{ Attitude: } \\
\hline Positive & 48 & 70.6 & 68 & 100 & 0.001 & 52 & 16.0 & 256 & 79.0 & 0.001 \\
\hline Negative & 20 & 29.4 & 0 & 0.0 & & 272 & 84.0 & 68 & 21.0 & \\
\hline \multicolumn{11}{|l|}{ Practice: } \\
\hline Good & 44 & 64.7 & 64 & 94.1 & 0.001 & 18 & 5.6 & 18 & 5.6 & 1000 \\
\hline Poor & 24 & 35.3 & 4 & 5.9 & & 306 & 94.4 & 306 & 94.4 & \\
\hline
\end{tabular}


Table (3): Association between socio-demographic data and KAP scores of security personnel and prisoners before application of health education program.

\begin{tabular}{|c|c|c|c|c|c|c|c|c|c|c|c|c|c|c|c|c|c|c|}
\hline \multirow{4}{*}{$\begin{array}{l}\text { Socio- } \\
\text { demographic } \\
\text { data }\end{array}$} & \multicolumn{18}{|c|}{ Pre-intervention KAP of security personnel $(\mathrm{N}=68)$} \\
\hline & \multicolumn{6}{|c|}{ Knowledge } & \multicolumn{6}{|c|}{ Attitude } & \multicolumn{6}{|c|}{ Practice } \\
\hline & \multicolumn{2}{|c|}{$\begin{array}{c}\text { Satis- } \\
\text { factory }\end{array}$} & \multicolumn{2}{|c|}{$\begin{array}{l}\text { Unsatis- } \\
\text { factory }\end{array}$} & \multirow{2}{*}{$\chi^{2}$} & \multirow{2}{*}{$\begin{array}{c}p- \\
\text { value }\end{array}$} & \multicolumn{2}{|c|}{ Positive } & \multicolumn{2}{|c|}{ Negative } & \multirow{2}{*}{$\chi^{2}$} & \multirow[b]{2}{*}{$\begin{array}{c}p- \\
\text { value }\end{array}$} & \multicolumn{2}{|c|}{ Good } & \multicolumn{2}{|c|}{ Poor } & \multirow{2}{*}{$\chi^{2}$} & \multirow{2}{*}{$\begin{array}{c}p- \\
\text { value }\end{array}$} \\
\hline & $\begin{array}{l}\mathrm{N} \\
(26)^{*} \\
(6)^{* *}\end{array}$ & $\%($ & $\begin{array}{c}\mathrm{N} \\
(42)^{*} \\
(318)^{* *}\end{array}$ & $\%$ & & & $\begin{array}{c}\mathrm{N} \\
(48)^{*} \\
(52)^{* *}\end{array}$ & $\%$ & $\begin{array}{c}\mathrm{N} \\
(20)^{*} \\
(271)^{* *}\end{array}$ & $\%$ & & & $\begin{array}{c}\mathrm{N} \\
(44) \\
(18)^{*}\end{array}$ & $\%$ & $\begin{array}{c}\mathrm{N} \\
(24) \\
(306)^{* *}\end{array}$ & $\%$ & & \\
\hline $\begin{array}{l}\text { Age: } \\
\quad<40 \text { years } \\
\quad \geq 40 \text { years }\end{array}$ & $\begin{array}{l}17 \\
9\end{array}$ & $\begin{array}{l}65.4 \\
34.6\end{array}$ & $\begin{array}{l}25 \\
17\end{array}$ & $\begin{array}{l}59.5 \\
40.5\end{array}$ & 0.23 & 0.63 & $\begin{array}{l}35 \\
13\end{array}$ & $\begin{array}{l}72.9 \\
27.1\end{array}$ & $\begin{array}{l}7 \\
13\end{array}$ & $\begin{array}{l}35.0 \\
65.0\end{array}$ & 8.59 & 0.003 & $\begin{array}{l}30 \\
14\end{array}$ & $\begin{array}{l}68.2 \\
31.8\end{array}$ & $\begin{array}{l}12 \\
12\end{array}$ & $\begin{array}{l}50.0 \\
50.0\end{array}$ & 2.17 & 0.14 \\
\hline $\begin{array}{l}\text { Sex: } \\
\quad \text { Male } \\
\text { Female }\end{array}$ & $\begin{array}{l}22 \\
4\end{array}$ & $\begin{array}{l}84.6 \\
15.4\end{array}$ & $\begin{array}{l}32 \\
10\end{array}$ & $\begin{array}{l}76.2 \\
23.8\end{array}$ & 0.79 & 0.41 & $\begin{array}{l}39 \\
9\end{array}$ & $\begin{array}{l}81.2 \\
18.8\end{array}$ & $\begin{array}{l}15 \\
5\end{array}$ & $\begin{array}{l}75.0 \\
25.0\end{array}$ & $0.34^{*}$ & 0.743 & $\begin{array}{l}36 \\
8\end{array}$ & $\begin{array}{l}81.8 \\
18.2\end{array}$ & $\begin{array}{l}18 \\
6\end{array}$ & $\begin{array}{l}75.0 \\
25.0\end{array}$ & $0.44 \#$ & 0.54 \\
\hline $\begin{array}{l}\text { Residence: } \\
\text { Rural } \\
\text { Urban }\end{array}$ & $\begin{array}{l}9 \\
17\end{array}$ & $\begin{array}{l}34.6 \\
65.3\end{array}$ & $\begin{array}{l}16 \\
26\end{array}$ & $\begin{array}{l}38.0 \\
61.9\end{array}$ & 0.08 & 0.77 & $\begin{array}{l}15 \\
33\end{array}$ & $\begin{array}{l}31.2 \\
68.8\end{array}$ & $\begin{array}{l}10 \\
10\end{array}$ & $\begin{array}{l}50.0 \\
50.0\end{array}$ & 2.14 & 0.144 & $\begin{array}{l}11 \\
33\end{array}$ & $\begin{array}{l}25.0 \\
75.0\end{array}$ & $\begin{array}{l}14 \\
10\end{array}$ & $\begin{array}{l}58.3 \\
41.7\end{array}$ & 7.42 & 0.006 \\
\hline & \multicolumn{18}{|c|}{ Pre-intervention KAP of Prisoners (N=324) } \\
\hline $\begin{array}{l}\text { Age: } \\
\quad<40 \text { years } \\
\quad \geq 40 \text { years }\end{array}$ & $\begin{array}{l}3 \\
3\end{array}$ & $\begin{array}{l}50.0 \\
50.0\end{array}$ & $\begin{array}{l}215 \\
103\end{array}$ & $\begin{array}{l}67.6 \\
32.4\end{array}$ & 0.83 & 0.39 & $\begin{array}{l}35 \\
17\end{array}$ & $\begin{array}{l}67.3 \\
32.7\end{array}$ & $\begin{array}{l}183 \\
89\end{array}$ & $\begin{array}{l}67.3 \\
32.7\end{array}$ & 0.00 & 0.97 & $\begin{array}{l}11 \\
7\end{array}$ & $\begin{array}{l}61.1 \\
38.9\end{array}$ & $\begin{array}{l}207 \\
99\end{array}$ & $\begin{array}{l}67.6 \\
32.4\end{array}$ & 0.33 & 0.566 \\
\hline $\begin{array}{l}\text { Sex: } \\
\quad \text { Male } \\
\text { Female }\end{array}$ & $\begin{array}{l}4 \\
2\end{array}$ & $\begin{array}{l}66.7 \\
33.3\end{array}$ & $\begin{array}{l}242 \\
76\end{array}$ & $\begin{array}{l}76.1 \\
23.9\end{array}$ & 0.29 & 0.63 & $\begin{array}{l}36 \\
16\end{array}$ & $\begin{array}{l}69.2 \\
30.8\end{array}$ & $\begin{array}{l}210 \\
62\end{array}$ & $\begin{array}{l}77.2 \\
22.8\end{array}$ & 1.52 & 0.22 & $\begin{array}{l}12 \\
7\end{array}$ & $\begin{array}{l}66.7 \\
33.3\end{array}$ & $\begin{array}{l}234 \\
72\end{array}$ & $\begin{array}{l}76.5 \\
23.5\end{array}$ & 0.84 & 0.394 \\
\hline $\begin{array}{l}\text { Education lev } \\
\text { Educated } \\
\text { Illiterate }\end{array}$ & $\begin{array}{l}3 \\
3\end{array}$ & $\begin{array}{l}50.0 \\
50.0\end{array}$ & $\begin{array}{l}104 \\
214\end{array}$ & $\begin{array}{l}32.7 \\
67.3\end{array}$ & 0.89 & 0.40 & $\begin{array}{l}36 \\
16\end{array}$ & $\begin{array}{l}69.2 \\
30.8\end{array}$ & $\begin{array}{l}181 \\
91\end{array}$ & $\begin{array}{l}66.5 \\
33.5\end{array}$ & 0.14 & 0.71 & $\begin{array}{l}3 \\
15\end{array}$ & $\begin{array}{l}16.7 \\
83.3\end{array}$ & $\begin{array}{l}104 \\
202\end{array}$ & $\begin{array}{l}34.0 \\
66.0\end{array}$ & 2.31 & 0.129 \\
\hline $\begin{array}{c}\text { Imprisonment } \\
<10 \text { years } \\
\geq 10 \text { years }\end{array}$ & $\begin{array}{l}5 \\
1\end{array}$ & $\begin{array}{l}83.3 \\
16.7\end{array}$ & $\begin{array}{l}266 \\
52\end{array}$ & $\begin{array}{l}83.6 \\
16.4\end{array}$ & 0.00 & 1.0 & $\begin{array}{l}44 \\
8\end{array}$ & $\begin{array}{l}84.6 \\
15.3\end{array}$ & $\begin{array}{l}227 \\
45\end{array}$ & $\begin{array}{l}83.4 \\
16.5\end{array}$ & 0.04 & 0.84 & $\begin{array}{l}15 \\
3\end{array}$ & $\begin{array}{l}83.3 \\
16.7\end{array}$ & $\begin{array}{l}256 \\
50\end{array}$ & $\begin{array}{l}81.2 \\
15.8\end{array}$ & 0.00 & 1.00 \\
\hline
\end{tabular}

Table (4): Association between socio-demographic data and KAP scores of security personnel and prisoners after application of health education program.

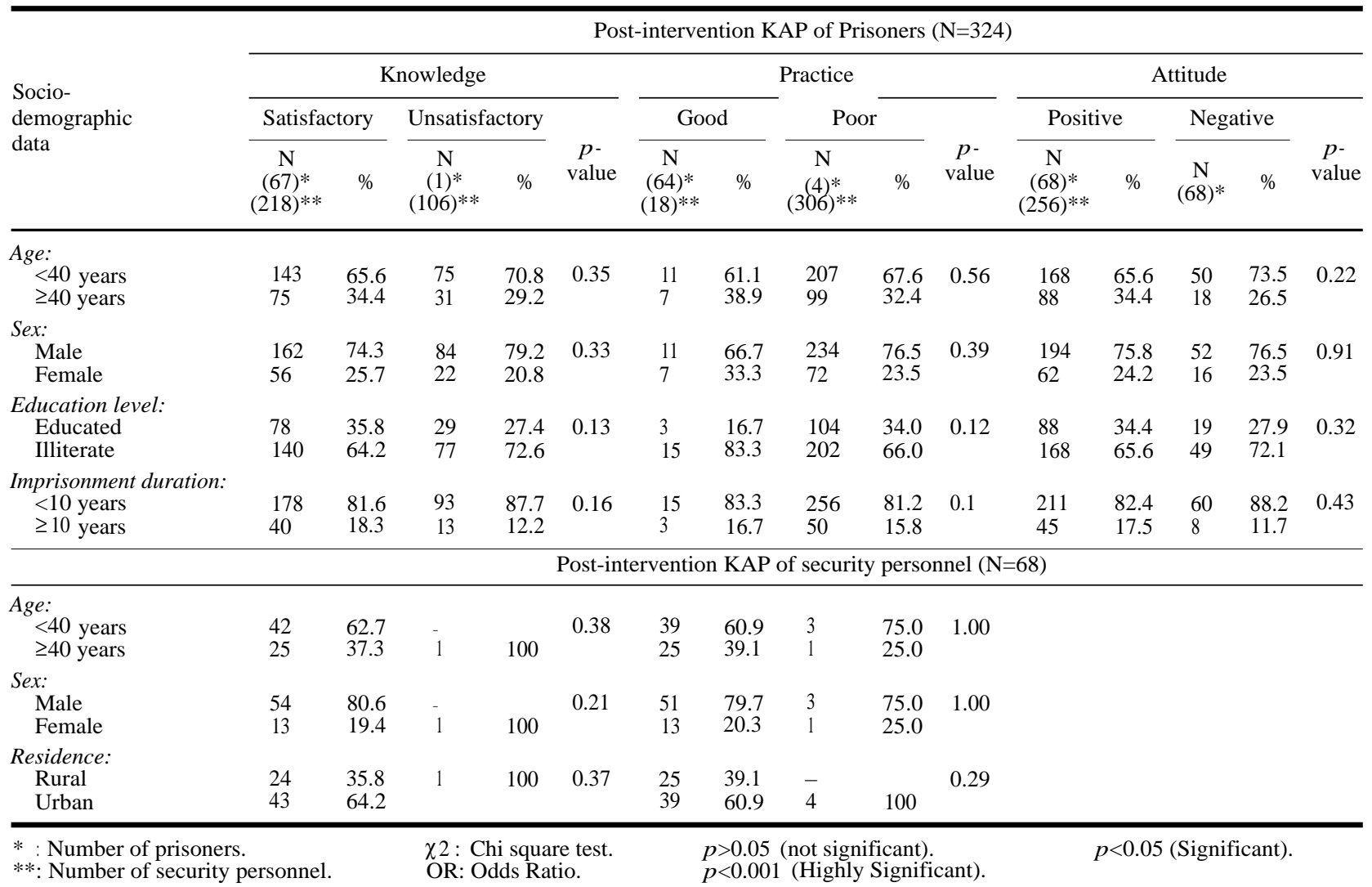


Table (5): Comparison between HBV and or HCV positive and negative studied groupsregarding preimplementation knowledge, attitude and practice.

\begin{tabular}{|c|c|c|c|c|c|c|c|c|c|c|c|c|}
\hline \multirow{3}{*}{ KAP } & \multicolumn{6}{|c|}{ Security personnel $(\mathrm{N}=68)$} & \multicolumn{6}{|c|}{ The studied prisoners $(\mathrm{N}=324)$} \\
\hline & \multicolumn{2}{|c|}{$\begin{array}{l}\text { Hepatitis } \\
\text { positive }\end{array}$} & \multicolumn{2}{|c|}{$\begin{array}{c}\text { Hepatitis } \\
\text { negative }\end{array}$} & \multirow{2}{*}{$x^{2}$} & \multirow{2}{*}{$\begin{array}{c}p- \\
\text { value }\end{array}$} & \multicolumn{2}{|c|}{$\begin{array}{l}\text { Hepatitis } \\
\text { positive }\end{array}$} & \multicolumn{2}{|c|}{$\begin{array}{l}\text { Hepatitis } \\
\text { negative }\end{array}$} & \multirow[t]{2}{*}{$x^{2}$} & \multirow{2}{*}{$\begin{array}{c}p- \\
\text { value }\end{array}$} \\
\hline & $\mathrm{N}(9)$ & $\%$ & $\mathrm{~N}(59)$ & $\%$ & & & $N(85)$ & $\%$ & N (239) & $\%$ & & \\
\hline \multicolumn{13}{|l|}{ Knowledge: } \\
\hline Satisfactory & 4 & 44.4 & 22 & 37.3 & $0.17 *$ & 0.72 & 6 & 7.1 & & & $17.19^{*}$ & 0.001 \\
\hline Unsatisfactory & 5 & 55.6 & 37 & 62.7 & & & 79 & 92.9 & 239 & 100 & & \\
\hline \multicolumn{13}{|l|}{ Attitude: } \\
\hline Positive & 5 & 55.6 & 43 & 72.9 & $1.13^{*}$ & 0.43 & 15 & 17.6 & 37 & 15.5 & 0.22 & 0.64 \\
\hline Negative & 4 & 44.4 & 16 & 27.1 & & & 70 & 82.4 & 202 & 84.5 & & \\
\hline \multicolumn{13}{|l|}{ Practice: } \\
\hline Good & 4 & 44.4 & 40 & 67.8 & $1.87 *$ & 0.26 & 2 & 2.4 & 16 & 6.7 & $2.25^{*}$ & 0.173 \\
\hline Poor & 5 & 55.6 & 19 & 32.2 & & & 83 & 97.6 & 223 & 93.3 & & \\
\hline $\begin{array}{l}\chi 2 \text { : Chi square te } \\
\text { OR: Odds Ratio. }\end{array}$ & & & $\begin{array}{l}*: \mathrm{Fi} \\
p>0 .\end{array}$ & $05(\mathrm{r}$ & Exa & $\begin{array}{l}\text { est. } \\
\text { ficant). }\end{array}$ & & & $p<0.001$ & $\begin{array}{l}\text { ignifi } \\
\text { (Highl }\end{array}$ & $\begin{array}{l}\text { cant). } \\
\text { ly Signi }\end{array}$ & cant). \\
\hline
\end{tabular}

\section{Discussion}

The current study examined KAP of HBV and $\mathrm{HCV}$ infection among security personnel and prisoners in Shebin El-Kom Public Prison (Menoufia Governorate, Egypt) before and after educational intervention. Owing to the information campaign, health education in community, and the public's increasing contact in daily life with people infected with HCV \& HBV, people would become more knowledgeable about HCV and HBV [17]. This study reported that the main source of information about HBV \& HCV was mass media in the form of TV (44.5\%) followed by health services (24.8\%), then from others who included family members, relatives, work mates, neighbors, friends $(24.2 \%)$. This was in agreement with what reported by several investigators [18-20]. We are reporting low pre-intervention knowledge levels among both security personnel and the studied prisoners (38.2\%, $1.9 \%$ respectively). Common methods of HBV \& $\mathrm{HCV}$ transmission, clinical picture and preventive and controlmeasures acknowledged by the studied security personnel and prisoners before educational intervention were limited $(39.7 \%, 29.4 \%, 39.7 \%$ and $3.7 \%, 4 \%, 3.7 \%$ respectively). These results were in accordance with Al-Thaqafy et al., [21] who reported that only $35.2 \%$ of Saudi National Guard personnel had some knowledge of HBV. Those who correctly identified the infectious nature of HBV increased were $33.3 \%$. This finding also were in consistent with Lambert and Paoline [22] who found that only $8 \%$ of informants felt officers were adequately informed about dealing with prisoners with infectious disease. Also these results were comparable to those reported in several study conducted on other Egyptian population sectors [23-26]. However these results were lower than those reported in by Badr et al., [27] who found that $55.2 \%$ of the secondary school students in one educational sector in Alexandria had fair levels of knowledge (50-75\%) about HBV. In the current work, there was no significant difference between males and females participants regarding their total knowledge score about hepatitis B and C. Similar to this study, gender was not found to be a significant risk factor for knowledge of hepatitis in studies carried out in Egypt and the United States $[28,29]$. However on the contrary to this, Batholomew [30] found that there was a significant difference between female and male students in both rural and urban settings concerning their knowledge about types of HBV and the different modes of transmission.In this study, there was no significant difference between age of the studied groups, his/her level of education, and residence and their total knowledge score about hepatitis B and C. On the contrary to this, Cheung et al., [31] in Colombia showed that higher HBV knowledge was significantly associated with increasing age $(p<0.001)$ and higher education $(p<0.001)$. In the current work, there wassignificant difference between practice of security personnel and their residence as those living in urban areas had better practice than living in rural areas. This explained by being most of security personals living in urban areas were policemen and doctors with high level of education and socioeconomic level while the majority of those living in rural areas were soldiers and jailers with lower level of education (mostly primary).

The study results showed marked improvement of overall KAP scores after educational intervention. This result agreed with the results of AlThaqafy et al., [21] who showed marked improve- 
ment of overall KAP scores and their components' scores regarding hepatitis B viral infection after educational intervention. This improvement was irrespective to their socio-demographic data. Similarly, a marked improvement (from 24\% to 84\%) in knowledge of hepatitis $\mathrm{C}$ transmission was observed in secondary school students in France after an educational slide show [32].Additionally Ingrand et al., [33] agreed with these results who found that knowledge about the transmission and availability of vaccine significantly improved when measured two months after an information session, suggesting that subsequent at-risk behaviors might be reduced. However, against these results, Cook and Bellis [34] reported that increases in knowledge do not always lead to improvement in behavior. In the current work, there were no significant differences in knowledge, attitude and practice before health education program among HBV and HCV positive and negative security personals. However, HBV \& HCV infected prisoners $(p<0.001)$ were significantly more knowledgeable about these viruses before educational intervention. $(p<0.001)$. This difference could be explained that infected prisoners visit doctors frequently, having more health awareness and being alert to any information about his disease.

\section{Conclusions:}

Based on the findings of the present study, we can conclude that, there were low levels of HBV \& HCV awareness among the studied security personnel and prisoners in Shebin El-Kom Public Prison (Menoufia Governorate, Egypt) before educational intervention. However there was significant improvement in all KAP scores level among security personnel and there was significant improvement in knowledge and attitude of prisoners without improvement in their practice score after application of health education program. Also, this study explored the potential role of mass media especially television as a good tool for conveying the proposed health education message. This indicated that heath education in its different forms and formal education is the important cornerstone in building up health awareness in our community.

\section{References}

1- TAIWO O.O. and BUKAR A.: Knowledge and attitude of prisoners towards HIV/AIDS infection," Research Center for Oral Health Research and Training Initiative for Africa, Vol. 1, No. 1, pp. 31-4, 2006.

2- TALAAT M., RADWAN E., EL-SAYED N., ISMAEL T., HAJJEH R. and MAHONEY F.J.: Case-control study to evaluate risk factors for acute hepatitis B virus infection in Egypt. Eastern Mediterranean Health Journal, 16 (1): $4-9,2010$.
3- GUERRA M., GARENNE M., MOHAMED M.K. and FONTANET A.: HCV burden of infection in Egypt: Results from a nationwide survey." J. Viral. Hepat., Vol. 12, No. 2, pp: 560-7, 2012.

4- EL-ZANATY and ANN WAY: Egypt Demographic and Health Survey." Cairo, Egypt: Ministry of Health, ElZanaty and Associates, and Macro International, 2009.

5- Center of Disease Control and Prevention (CDC MMWR): Recommendations for Identification and Public Health Management of Persons with Chronic Hepatitis B Virus Infection". Recommendations and reports, 57 (RR08); Pp. 1-20, 2008.

6- SENOK A.C. and BOTTA G.A.: Human immunodeficiency virus and hepatitis virus infection in correctional institutions in Africa: Is this the neglected source of an epidemic? J. Med. Microbiol., 55: 481-2. [PubMed], 2006.

7- STARK K., HERRMANN U., EHRHARDT S. and BIENZLE U.: A syringe exchange programme in prison as prevention strategy against HIV infection and hepatitis $\mathrm{B}$ and $\mathrm{C}$ in Berlin, Germany". Epidemiology and Infection, August. 134 (4): 8149. Doi: 10.1017/S0950268805005613. PMC 2870452. PMID 1637118, 2006.

8- BUTLER T. and PAPANASTASIU C.: National prison entrants' blood borne virus and risk behavior survey report-2004 and 2007: Prevalence of HIV, hepatitis C, hepatitis B, and risk behaviors among Australian prison entrants (National Drug Research Institute, National Centre in HIV Epidemiology and Clinical Research, Sydney, 2008).

9- MAHFOUD Z., KASSAK K., KREIDIEH K., SHAMRA S. and RAMIA S.: Prevalence of antibodies to Human Immunodeficiency Virus (HIV), hepatitis B and hepatitis $\mathrm{C}$ and risk factors in prisoners in Lebanon J. Infect. Dev. Ctries., pp. 144-9, 2010.

10- ALLWRIGHT S., BRADLEY F., LONG J., BARRY J., THORNTON L. and PARRY J.V.: Prevalence of antibodies to hepatitis B, hepatitis C, and HIV and risk factors in Irish prisoners: Results of a national cross sectional survey. B.M.J., 321: 78-82, 2000.

11- HELLARD M.E., AITKEN C.K. and HOCKING J.S . Tattooing in prisons not just a pretty picture. Am. J. Infect. Control., 35/477, 2007.

12- EL-BELTAGY K.E., AL-BALAWI I.A., ALMUNEEF M. and MEMISH Z.A.: Prevalence of hepatitis B virus markers among blood donors in a tertiary hospital in Tabuk, northwestern Saudi Arabia. Int. J. Infect. Dis., 12 (5): 495-9, 2008.

13- ZAHROTUR R. HINDUAN, HARRY SUHERMAN, W.J. LUCAS PINXTEN, BACHTIALISJAHBANA and HARM J.: HIV-related knowledge and attitudes among Indonesian prison officers International Journal of Prisoner Health J. Vol. 9 No. 2, pp. 92-102, Q Emerald Group Publishing Limited, ISSN 1744-9200. Doi 10.1108/ 174492013 11326961, 2013.

14- STEPHANIE E., PERRETT M.E. and MARION L.: Evaluation of an e-learning package to improve understanding of blood-borne viruses amongst prison staff in Wales, Ukinternational Journal of Prisoner Healthdoi 10.1108/Ijph-04-2013-0019 Vol. 10 No. 1, pp. 47-54, C Emerald Group Publishing Limited, ISSN 1744-9200, 2014. 
15- ROYA M., FARAHNAZ J., FATEMEH SOUTI and ZAHRA ATRKAR-ROUSHAN: Knowledge and attitude of medical science students toward hepatitis B and C infections." Int. J. Clin. Exp. Med., 6 (3): 197-205 www.ijcem.com/ISSN:1940-5901/IJCEM1301003. 2013.

16-GREENBERG J.S.: Health education and health promotion. 5th edition. New York: McGraw-Hill, 2004.

17- MARÍA T.M.S., SANDIE M., GABRIELLE M., EMILIE P., LAURENCE S. and JEAN-PIERRE V.: Changes in French people's misconceptions about hepatitis C. Preventive Medicine, 42 (2): 150-3, 2006.

18- ALAM M.W.Z.: Knowledge, attitudes and practices about Hepatitis B and C among young healthy male Maqbool Pakistan, 17 (4): 147-50, 2006.

19- CHEN J., SMITH B.J., LOVEDAY S., BAUMAN A., COSTELLO M., MACKIE B., et al.: Impact of a mass media campaign upon calls to the New South Wales Hep. C. helpline, 16 (1): 11-4, 2005.

20- SMITH B.J., BAUMAN A.E., CHEN J., LOVEDAY S., COSTELlO M., MACKIE B., et al.: Hepatitis C in Australia: Impact of a assess education campaign. Am. J. Prev. Med., 31 (6): 492-8, 2006.

21- AL-THAQAFY M.S., HANAN H. BALKHY, ZIAD M., YAHYA M., ADEL I., et al.: Improvement of the low knowledge, attitude and practice of hepatitis B virus infection among Saudi national guard personnel after educational intervention." B.M.C. Research Notes, 5: 597 http://www.biomedcentral.com/1756-0500/5/597, 2012.

22- LAMBERT E.G. and PAOLINE E.A.: "The impact of medical issues on the job stress and job satisfaction of jail staff", Punishment and Society, Vol.: 7 No. 3, pp. 259-75, 2005

23- SALEH A.D., AMR S., IRENE A., JUDY H. and CHRISTOPHER A.: Knowledge and perceptions of hepatitis C infection and pesticides use in two rural villages in Egypt.". B.M.C. Public Health, 14: 501 http://www.biomedcentral. com/1471-2458/14/501, 2014.

24- EASSA S., EISSA M., SAMAR M., MOHAMED H. and IBRAHIM M.: Prevalence of Hepatitis C Virus Infection and Evaluation of a Health Education Program in El-
Ghar Village in Zagazig, Egypt." J. Egypt Public Health Assoc., Vol. 82 No. 5 \& 6, 2007.

25- ABDEL-MOHSEN A.M., SAMI S.M., ELSOROUGY S.M., HASSANIN A.I. and SHAABAN F.A.: Knowledge and behavior towards viral hepatitis among Egyptian children, their mothers and pediatricians." Med. J. Cairo Univ., 74 (2): 275-81, 2006

26- SAMI M.S., IMAN I. SALAMA, DALIA M. ELMOSALAMI, GHADA. ABDEL-LATIF and AMANY T. ABDEL HAMID: Knowledge of and risky behaviours towards hepatitis B virus infection among Egyptian school children." Arab Journal of Gastroenterology, 16: 94-8, 2015.

27- BADR H.H., MOUNIR G.M. and MAHDY N.H.: Measurement of KAP concerning hepatitis B among secondary school students in Alexandria." J. Egypt Public Health Assoc., 74 (3-4): 407-37, 1999.

28- WIECHA J.M.: Differences in knowledge of hepatitis B among Vietnamese, African-American, Hispanic, and white adolescents in Worcester, Massachusetts." Pediatrics, 104 (5): 1212-6, 1999.

29- BATHOLOMEW C.: Knowledge, attitude and practices (KAP) concerning hepatitis B among adolescents in the Upper West Region of Ghana. Rural Urban Gradient, 159, 2011.

30- CHUNG P.W., SUEN S.H., CHAN O.K., LAO T.H. and LEUNG T.Y.: Awareness and knowledge of hepatitis B infection and prevention and the use of hepatitis B vaccination in the Hong Kong adult Chinese population. Chin. Med. J. (Engl). Feb., 125 (3): 422-7, 2012.

31- GARDELLA F., MARINE-BARJOAN E., TRUCHI R., FODELLA L., DELASALLE P.,SATTONNET C., et al.: Hepatitis $\mathrm{C}$ awareness among adolescents in the AlpesMaritimes area of France". Gastroenterol. Clin. Biol., 31 (5): 485-92, 2007.

32- INGRAND I., VERNEAU A., SILVAIN C. and BEAUCHANT M.: Prevention of viral hepatitis C: Assessment of a comic strip-based information." Eur. J. Public Health, 14 (2): 147-3, 2004.

33- COOK P.A. and BELLIS M.A.: Knowing the risk: Relationships between risk behavior and health knowledge". Public Health, 115 (1): 54-61, 2001. 


\section{فاعلية البرثامج التثقيفى وتآثيره على معارف وإتجاهات وممارسات

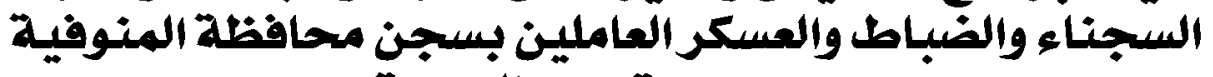

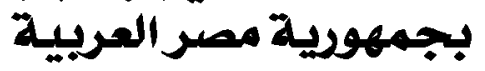

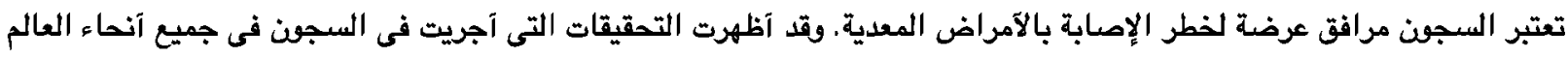

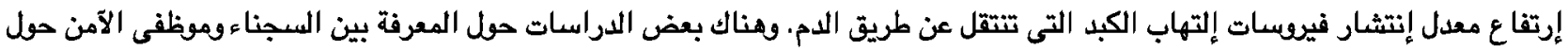

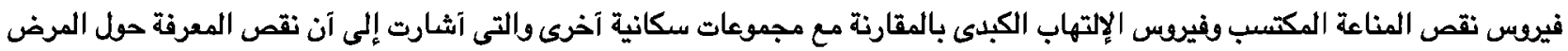

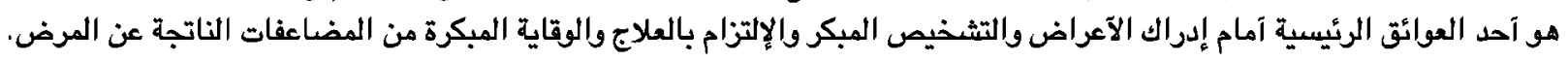

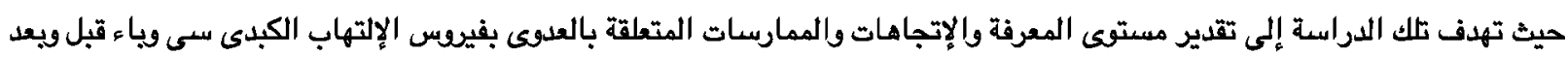

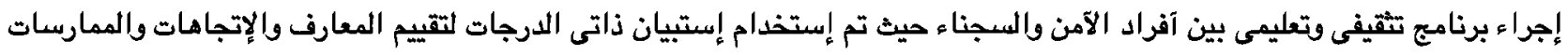

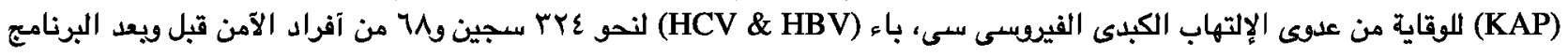

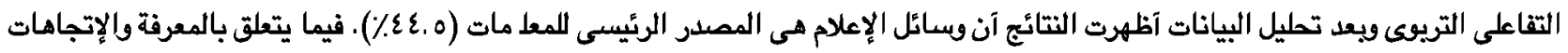

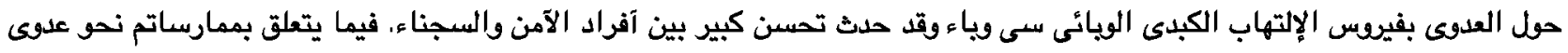

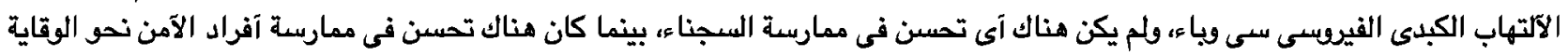

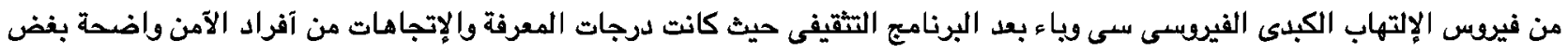

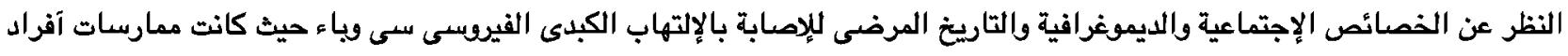

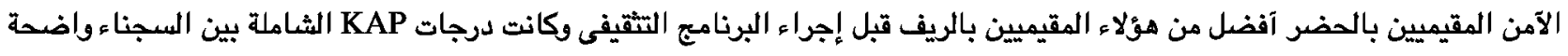

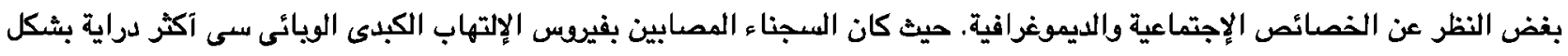

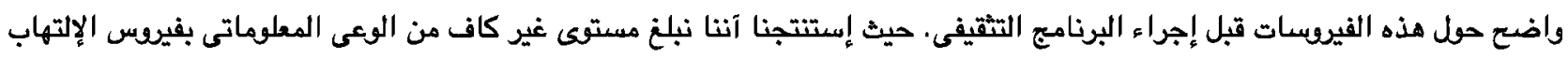

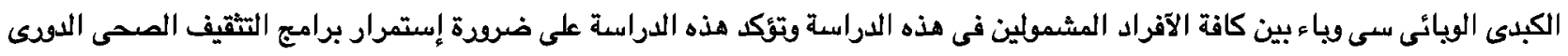

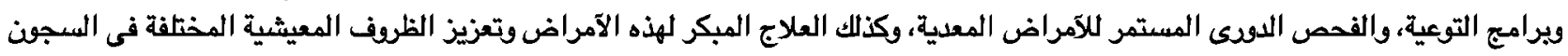

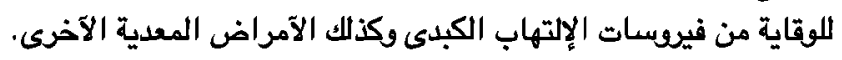

